\title{
Massive Pediatric Facial Plexiform Neurofibromatosis in Iraq
}

\author{
Tahrir N. Aldelaimi ${ }^{1 *}$ and Afrah A. Khalil ${ }^{2}$ \\ ${ }^{1}$ Department of Oral \& Maxillofacial Surgery, College of Dentistry, Anbar University, Anbar Province, Iraq \\ ${ }^{2}$ Specialist Dentist, Dental Health Department, Anbar Health Directorate, Ministry of Health, Iraq
}

Received: November 09, 2013; Accepted: December 18, 2013; Published: December 19, 2013

"Corresponding author: Tahrir N. Aldelaimi, Department of Oral \& Maxillofacial Surgery, College of Dentistry, Anbar University, Ramadi city, Anbar Province, Iraq, Tel: 009-647814050913; E-mail: tahrir_aldelaimi@yahoo.com

Keywords: Plexiform neurofibroma; Neurological abnormalities; Facial mass; Neurofibromatosis

\section{Introduction}

Plexiform Neurofibroma or Pachydermatocoele (PN/P) is a type of neurofibromatosis caused by excessive growth of the neural tissue in the subcutaneous fat and can be seen in connection with the branches of trigeminal nerve [1] in maxillofacial region. In contrast It has also been reported in retroperitoneal region, anterior abdominal wall, paraspinal and mediastinal area [2,3]. Plexiform neurofibroma or pachydermatocoele (PN/P) is diffuse, elongated fibromas coursing along the nerves, they are mostly involve the trigeminal or upper cervical nerves and early in life that has an overgrowth of epidermal and subcutaneous tissue associated with a wrinkled and pendulous appearance $[4,5]$. Plexiform neurofibroma or pachydermatocoele (PN/P) need to be monitored frequently because of incidence of development into malignant peripheral nerve sheath tumors. Other complications include bleeding from trauma, neurological deficits, limited limb, and psychological disturbance because of abnormal anatomy $[6,7]$.

\section{Case Report}

A 10-year-old female patient presented to Maxillofacial Surgery Department at Ramadi Teaching Hospital, Anbar Province, Iraq during last July 2012 with a history of gradual swelling since 12 months. There was no history of any similar swelling elsewhere over the body. There was no history of any pain, trauma or constitutional symptoms or any distal neurological deficit. She had neither history of any bowel or bladder complaints nor family history. Clinical and CT examination was done, consultation with neurosurgeon and ophthalmologist for proper evaluation and assessment and a careful histopathological evaluation of the specimen is necessary. On clinical examination, there were small cafe-au-lait spots on side of face, Ophthalmic, ENT and neurosurgical examinations were normal and systemic examination revealed no neurological deficit. The massive growth of plexiform neurofibroma or pachydermatocoele (PN/P) was involving the right maxillary sinus extended down to hard palate but not exceeding the midline, anteriorly the growth causing facial asymmetry. Upward direct extension to orbit to retrobulbar area ending with proptosis. Posteriorly; the growth reaching the pterygo-palatine fissure and posterior maxilla. Chest radiograph and Ultrasonography of the abdomen revealed no intra-abdominal abnormality. Wide excision of the mass and split skin grafting of the raw area was done. Histopathology confirmed the diagnosis was done (Figures 1-4).

\section{Discussion}

Neurofibroma is seen either as a solitary lesion or as part of the generalized syndrome of neurofibromatosis (usually neurofibromatosis type 1 [NF-1], also called von Recklinghausen disease of the skin). The solitary form does not differ from the disseminated form or the multiple form of the disease, except that systemic and hereditary factors present in the disseminated form are absent in the solitary type. Multiple neurofibromas have also been strongly associated with the polyglandular syndrome multiple endocrine neoplasia type 3. Oral and maxillofacial region involvement by a solitary and peripheral plexiform neurofibroma in patients with no other signs of neurofibromatosis

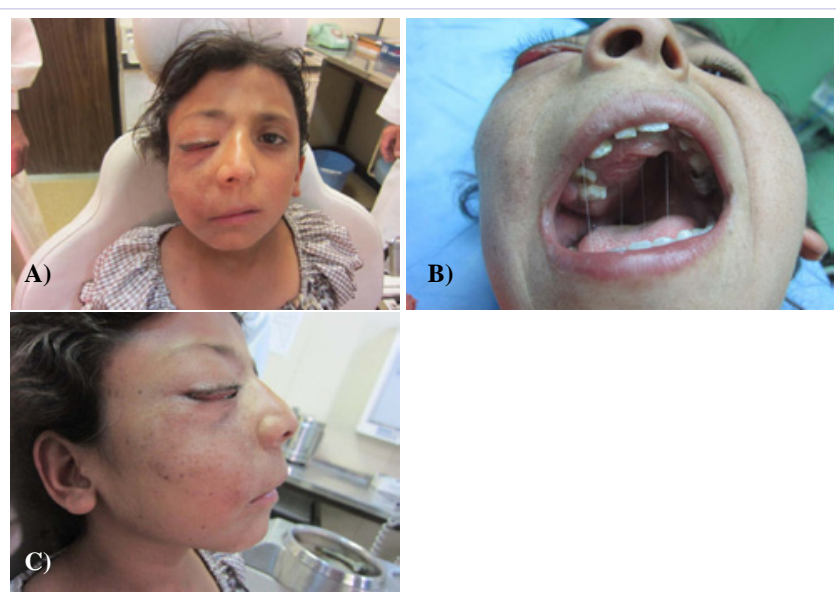

Figure 1: Preoperative view (frontal, intraoral and side view). 
is uncommon. Sporadic cases have been reported in the submandibular gland, tongue, and on the periosteum at the mental foramen. This sporadic syndromic occurrence has also been seen in the cutaneous region, and several authors have suggested that these isolated neurofibromas may represent a hamartomatous growth. The World Health Organization (WHO) has subdivided neurofibromas into 2 broad categories: dermal and plexiform. Dermal neurofibromas arise from a single peripheral nerve, while plexiform neurofibromas are associated with multiple nerve bundles. Other clinicopathologic subtypes include localized neurofibroma (sporadic neurofibroma), diffuse neurofibroma, plexiform neurofibroma, and epithelioid neurofibroma. These are benign, slow-growing nerve tumors, similar to schwannomas. They commonly occur in patients who suffer from neurofibromatosis, a genetic disorder resulting in

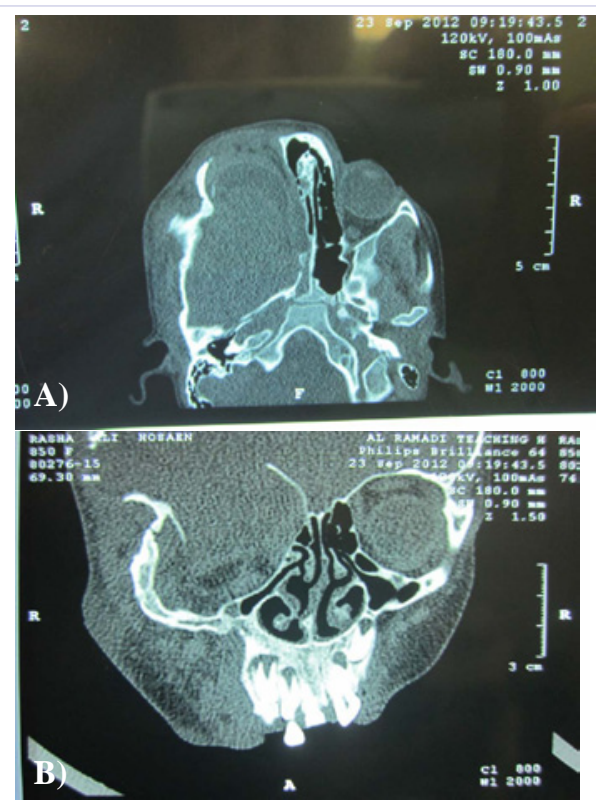

Figure 2: A) Preoperative coronal CT scan showing tumor extension that involving Rt orbit and maxillary sinus. B) Preoperative axial CT scan that extension of tumor that involves the orbit and maxillary sinus and extended posteriorly to base of skull.

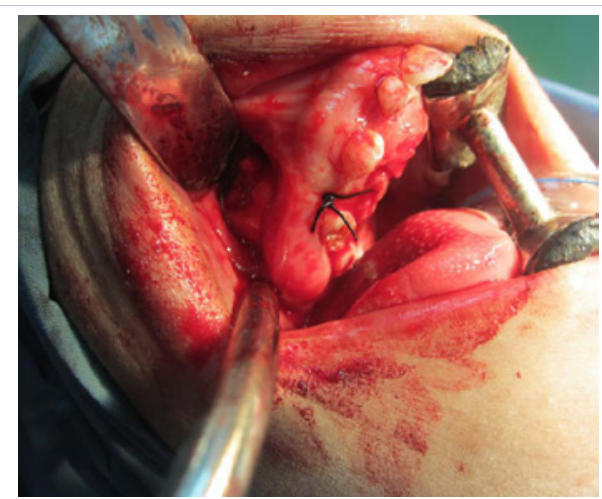

Figure 3: Intraoperative view showing incisional biopsy taking procedure.

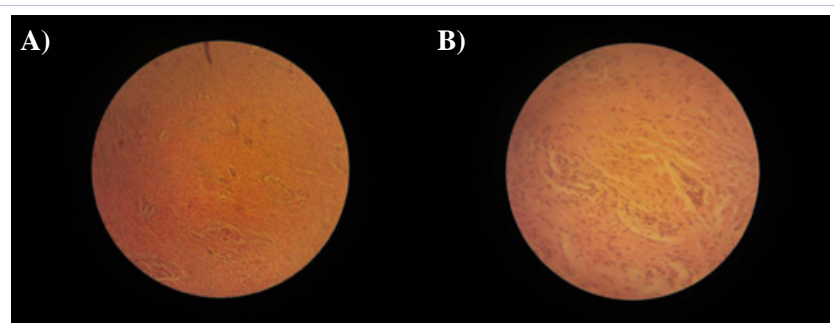

Figure 4: $\mathrm{H} \& \mathrm{E}$ (10X and 40X respectively) showing lobular proliferation of spindle cells in a loose fibular background.

multiple tumors throughout the body. Plexiform neurofibroma or pachydermatocoele (PN/P) considered as a type of generalised neurofibromatosis that was occurs due to overgrowth of neural tissue in the subcutaneous fat. It is also considered as a hamartoma than a typical tumour [1,6]. It is a type of neurofibroma manifesting as a diffuse overgrowth of subcutaneous tissue, usually involving the face, scalp, neck, and chest but occasionally occurring in the abdomen or pelvis. The tumors tend to progress, and may extend along nerve roots to eventually involve the spinal roots and spinal cord. Neurological abnormalities are uncommon. These tumours could beundergone malignant change in about $2.4-5 \%$ of the cases $[7,8]$. It is commonly seen along the distribution of trigeminal nervein maxillofacial region, however; isolated cases of segmental plexiform neurofibromas have also been reported in other parts of the body including scalp, neck, chest, pelvis and few cases in the abdomen $[3,5,9]$. Malignancies are usually large, irregular, infiltrative, and necrotic with heterogeneous contrast enhancement and hence contrast enhanced computed tomography is useful in predicting resectability, detecting metastasis, and evaluating response of the treatment $[7,8]$. Therefore; incisional tissue biopsy in such cases would yield a histopathological confirmation [5,6] and in turn plexiform neurofibromas need to be monitored frequently because of development into malignant peripheral nerve sheath tumors. Other complications include bleeding from trauma, neurological deficits and psychological disturbance because of abnormal anatomy. In each of the above complications surgery might be helpful. However, surgeons should always keep in mind that surgery for plexiform neurofibromas patient has an extreme difficult and delicate surgical procedures and in turn good patient preparation, care and caution should be experienced during surgery $[9,10]$.

\section{References}

1. Sengupta, S. P. (1996). Tumours and cysts. In long and short cases in surgery. (1st ed., pp. 39-75). Calcutta: New Centre Book Agency Publications.

2. Aloi, F. G., Massobrio. R. (1989). Solitary plexiform neurofibroma. Dermatologica, 179, 84-6.

3. Som. A. (1985). Tumours and Cysts of Skin and subcutaneous tissue. In Principles and Practice of Modern Surgery. (5th ed., pp. 70-81). Calcutta: PS Publishers.

4. Littlewood. A. H., Stilwell. J. H. (1983). The vascular features of 
plexiform neurofibroma with some observations on the importance of pre-operative angiography and the value of pre-operative intraarterial embolisation. BrJ Plast Surg, 36, 501-506.

5. Calzavara. P. G., Carlino. A., Anzola. G. P., \& Pasolini. M. P. (1988). Segmental neurofibromatosis. Case report and review of the literature. Neurofibromatosis, 1, 318-22.

6. Harper. J. I. (1998). Genetics and genodermatoses. In R. H. Champion, J. L. Burton, D. A. Burns, S. M. Breathnach (Eds.), Text book of Dermatology (6th ed., pp. 378-84). Oxford: Blackwell Science.

7. Pui, M. H., Yang, Z. Y., \& Li, Z. P. (1998). Computed tomography of abdominal neurogenic tumours. Australasian Radiology, 42, 183-7.

8. Levine, E., Huntrakoon, M., \& Wetzel, L. H. (1987). Malignant nerve sheath neoplasms in neurofibromatosis: Distinction from Benign tumours by using imaging techniques. Am J Roentegenol, 149, 1059-64.

9. Darie, H., Veran, Y., Guyadec, T., Gros, P., \& Millet, P. (1998). Cutaneous splanchnic neurofibromatosis. Ann Dermatol Venerol, 125(8), 509-11.

10. Pivnick, E. K., Riccardi, V. M. (1999). The neurofibromatoses. In I. M. Freedberg, A. Z. Eisen, K. Wolff, K. F. Austen, Goldsmith LA, Kartz SI, Fitzpatrick TB (Eds.), Fitzpatrick's Dermatology in General Medicine(pp. 2152-58). New York: Mc graw Hill. 